J. Nonlinear Var. Anal. 3 (2019), No. 2, pp. 127-140

Available online at http://jnva.biemdas.com

https://doi.org/10.23952/jnva.3.2019.2.02

\title{
ITERATIVE ALGORITHMS FOR SPLIT VARIATIONAL INEQUALITIES AND GENERALIZED SPLIT FEASIBILITY PROBLEMS WITH APPLICATIONS
}

\author{
CHARLES E. CHIDUME*, MONDAY O. NNAKWE ${ }^{1}$ \\ African University of Science and Technology, Abuja, Nigeria
}

\begin{abstract}
In this paper, we present extragradient and modified extragradient iterative algorithms for solving a common solution of a finite family of split variational inequalities and generalized split feasibility problems. Applications of our main results to equilibrium and optimization problems are provided. A numerical experiment is provided to illustrate the convergence of the sequences generated in our proposed algorithms.
\end{abstract}

Keywords. Equilibrium and optimization problems; Monotone mappings; Nonexpansive mappings; Split variational inequalities; Strong convergence.

2010 Mathematics Subject Classification. 47H09, 47J25, 47J05.

\section{INTRODUCTION}

Let $M_{i}, i=1, \cdots, N$, and $Q_{j}, j=1, \cdots, p$, be nonempty closed and convex subsets of real Hilbert spaces $H_{1}$ and $H_{2}$, respectively such that $M=\cap_{i=1}^{N} M_{i} \neq \emptyset$ and $Q=\cap_{j=1}^{p} Q_{j} \neq \emptyset$. Let $G_{i}: H_{1} \rightarrow H_{1}, i=$ $1, \cdots, N$ and $A_{j}: H_{2} \rightarrow H_{2}, j=1, \cdots, p$ be given mappings and let $B$ be a bounded linear map from $H_{1}$ to $\mathrm{H}_{2}$.

The common solution of split variational inequality problem denoted by (CSSVIP) is to find an element $u^{*} \in M$ such that

$$
\begin{aligned}
& \left\langle u-u^{*}, G_{i}\left(u^{*}\right)\right\rangle \geq 0, \forall u \in M_{i}, i=1,2, \cdots, N, \text { and } \\
& v^{*}=B u^{*} \in Q \text { such that }\left\langle v-v^{*}, A_{j}\left(v^{*}\right)\right\rangle \geq 0, \forall v \in Q_{j}, j=1,2, \cdots, p .
\end{aligned}
$$

Let $\mathscr{D}$ be the solution set of the (CSSVIP) given by:

$$
\mathscr{D}=\left\{u^{*} \in \cap_{i=1}^{N} V I\left(M_{i}, G_{i}\right): B u^{*} \in \cap_{j=1}^{p} V I\left(Q_{j}, A_{j}\right)\right\} .
$$

We observe that $u^{*} \in(C S S V I P)$ if and only if $u^{*}=P_{M_{i}}\left(I-\mu B_{i}\right) u^{*}$, for each $i=1, \cdots, N$, such that $B u^{*}=P_{Q_{j}}\left(I-\gamma A_{j}\right) B u^{*}$ for each $j=1, \cdot, p$, where $P_{M_{i}}, P_{Q_{j}}$ are the metric projections of $M_{i}$ on $H_{1}$ and $Q_{j}$ on $H_{2}$, respectively, and $\mu>0$ and $\gamma>0$. Obviously, if $N=1$, then problem (1.1) is reduced to the well-known split variational inequality problem (SVIP), which was introduced by Censor, Gibali and Reich [5]. The motivation for studying problem (1.1) with $N>1$ stems from a simple observation that if we choose $G_{i} \equiv 0$, then the problem is reduced to finding $u^{*} \in \cap_{i=1}^{N} M_{i}$, which is the known convex

${ }^{*}$ Corresponding author.

E-mail addresses: cchidume@ aust.edu.ng (C.E. Chidume), mondaynnakwe@ gmail.com (M.O. Nnakwe).

Received October 29, 2018; Accepted March 14, 2019.

(C)2019 Journal of Nonlinear and Variational Analysis 
feasibility problem (CFP) that $B u^{*} \in \cap_{j=1}^{p} V I\left(Q_{j}, A_{j}\right)$. If the sets $M_{i}$ are the fixed point sets of mappings $S_{i}: H_{1} \rightarrow H_{1}$, then, the common feasibility problem, (CFP) is the common fixed points problem (CFPP) whose image under $B$ is a common solution to (CSVIP). If we choose $G_{i} \equiv 0$ and $A_{j} \equiv 0$, then problem (1.1) is reduced to finding $u^{*} \in \cap_{i=1}^{N} M_{i}$ such that $B u^{*} \in \cap_{j=1}^{p} Q_{j}$. This is the well known multiple-sets split feasibility problem which serves as a model for many inverse problems where constraints are imposed on the solutions in the domain of a linear operator as well as in the range of the operator.

In 1976, Korpelevich [11] proposed the following projection extragradient method in finite dimensional spaces

$$
\left\{\begin{array}{l}
x_{1} \in H, \\
y_{n}=P_{M}\left(x_{n}-\tau f\left(x_{n}\right)\right), \\
x_{n+1}=P_{M}\left(x_{n}-\tau f\left(y_{n}\right)\right),
\end{array}\right.
$$

for each $n \geq 1$. It was shown that the sequence converges to some point in $V I(f, M)$ if $f$ is monotone and $L$-Lipschitz.

In recent years, the extragradient method has received a lot of attention (see, e.g., [2, 6, 7, 10, 13, 14, 17] and the references therein). In 2006, Nadezhkina and Takahashi [15] introduced a hybrid extragradient method and proved a strong convergence theorem in a real Hilbert space. In 1994, Censor and Elfving [4] proposed a split feasibility problem (SFP) for modelling medical image reconstruction problems. Recently, this SFP has been extended to the intensity-modulation therapy treatment planning. The SFP is to find an element $u^{*} \in M$ satisfying the conditions:

$$
u^{*} \in M \text { and } B u^{*} \in Q,
$$

where $M$ and $Q$ are nonempty closed and convex subsets of real Hilbert spaces $H_{1}$ and $H_{2}$, respectively, and $B$ is a bounded linear mapping from $H_{1}$ to $H_{2}$. In 2010, Censor, Gibali and Reich [5] proposed a split variational inequality problem (SVIP), which is to find an element $u^{*} \in M$ satisfying the following conditions:

$$
u^{*} \in V I(M, G) \text { and } B u^{*} \in V I(Q, A),
$$

where $M$ and $Q$ are nonempty closed and convex subsets of real Hilbert spaces $H_{1}$ and $H_{2}$, respectively, $G$ is a mapping from $H_{1}$ to $H_{1}, A$ is a mapping from $H_{2}$ to $H_{2}$, and $B$ is a bounded linear mapping from $H_{1}$ to $H_{2}$. They proposed the multiple set split variational inequality problem: finding an element $u^{*} \in \cap_{i=1}^{N} M_{i}$ satisfying the following conditions:

$$
u^{*} \in \cap_{i=1}^{N} V I\left(M_{i}, G_{i}\right) \text { and } B u^{*} \in \cap_{j=1}^{p} V I\left(Q_{j}, A_{j}\right) .
$$

Recently, Tian and Jiang [19] studied the following algorithm for approximating a solution of the SFP in a real Hilbert space:

$$
\left\{\begin{array}{l}
x_{1}=x \in C, \\
y_{n}=P_{C}\left(x_{n}-\gamma_{n} A^{*}(I-T) A x_{n}\right), \\
t_{n}=P_{C}\left(y_{n}-\lambda_{n} f\left(y_{n}\right)\right) \\
x_{n+1}=P_{C}\left(y_{n}-\lambda_{n} f\left(t_{n}\right)\right), \forall n \geq 1,
\end{array}\right.
$$

where $\left\{\gamma_{n}\right\} \subset[a, b]$ for some $a, b \in\left(0, \frac{1}{\|A\|^{2}}\right)$, and $\left\{\lambda_{n}\right\} \subset[c, d]$ for some $c, d \in\left(0, \frac{1}{K}\right)$.

More precisely, they proved the following theorem. 
Theorem 1.1. Let $H_{1}$ and $H_{2}$ be real Hilbert spaces. Let $C$ be a nonempty closed and convex subset of $H_{1}$. Let $A: H_{1} \rightarrow H_{2}$ be a bounded linear mapping such that $A \neq 0, f: C \rightarrow H_{1}$ a monotone and $K$-Lipschitz continuous mapping and $\mathrm{T}: \mathrm{H}_{2} \rightarrow \mathrm{H}_{2}$ a nonexpansive mapping. Assume that $\Gamma=\{z \in V I(C, f): A z \in$ $F(T)\} \neq \emptyset$. Let $\left\{x_{n}\right\},\left\{y_{n}\right\}$ and $\left\{t_{n}\right\}$ be sequences generated by (1.4). Then, $\left\{x_{n}\right\}$ converges weakly to a point $z \in \Gamma$, where $z=\lim P_{\Gamma} x_{n}$.

Furthermore, they proved a weak convergence theorem for a split variational inequality problem in a real Hilbert.

In this paper, motivated by the results announced in Censor et al. [3], we propose an extragradient and a modified extragradient iterative algorithms, and prove strong convergence theorems in a real Hilbert space. Our theorems improve and complement the related results in Censor et al. [3], Tian and Jiang [19]. We present numerical experiments to illustrate the convergence of our algorithms. This paper is organized as follows. In Section 2, we present some preliminaries. In Section 3, we present our main convergence results. In Section 4, we give some applications of our main results. In Section 5, we present a numerical experiment to illustrate the convergence of our algorithms, and compare our theorems with some recent important results to conclude this paper.

\section{PRELIMINARIES}

Let $M$ be a nonempty closed and convex subset of a real Hilbert space $H$. We denote $v_{n} \rightarrow x^{*}$ and $v_{n} \rightarrow$ $x^{*}$ to indicate that a sequence $\left\{v_{n}\right\}$ converges weakly to $x^{*}$ and converges strongly to $x^{*}$, respectively, and use ism to stand for the inverse strongly monotone.

Lemma 2.1. (Goebel and Reich, [9]) Let M be a nonempty closed and convex subset of a real Hilbert space $H$. Then, the following hold.

(1) $\left\|v-P_{M} u\right\|^{2}+\left\|P_{M} u-u\right\|^{2} \leq\|v-u\|^{2}, \forall v \in M, u \in H$,

(2) $z=P_{M} u \Longleftrightarrow\langle z-v, u-z\rangle \geq 0, \forall v \in M$, where $P_{M}$ is the metric projection of $H$ onto $M$.

Definition 2.1. Let $T: H \rightarrow H$ be a mapping.

(1) $T$ is said to be $\alpha$-averaged if $T=(1-\alpha) I+\alpha S$, where $\alpha \in(0,1), S: H \rightarrow H$ is a nonexpansive mapping and $I$ is the identity mapping.

(2) $T$ is closed if for any sequence $\left\{v_{n}\right\} \subset H$ with $v_{n} \rightarrow x^{*}$ and $T v_{n} \rightarrow y$, then $T x^{*}=y$.

(3) $(I-T)$ is demi-closed at 0 if for any sequence $\left\{v_{n}\right\} \subset H$ with $v_{n} \rightarrow x^{*}$ and $v_{n}-T v_{n} \rightarrow 0$, then $T x^{*}=x^{*}$.

Remark 2.1. The set of fixed points of a mapping $T$ denoted by $F(T)$. If $T$ is nonexpansive, then $F(T)$ is closed and convex.

Definition 2.2. Let $B: H \rightarrow H$ be a mapping.

(1) $B$ is said to be monotone if, for each $u, v \in H,\langle u-v, B u-B v\rangle \geq 0$.

(2) $B$ is said to be $\eta$-ism with constant $\eta>0$ if, for each $u, v \in H$,

$$
\langle u-v, B u-B v\rangle \geq \eta\|B u-B v\|^{2} .
$$


Lemma 2.2. [20]

(1) $T$ is nonexpansive if and only if $(I-T)$ is $\frac{1}{2}-i s m$.

(2) If $T$ is $v$-ism and $\gamma>0$, then, $\gamma T$ is $\frac{v}{\gamma}$-ism.

(3) $T$ is averaged if and only if $(I-T)$ is $v$-ism for some $v>\frac{1}{2}$. Indeed, for $\eta \in(0,1), T$ is $\eta$-averaged if and only if $(I-T)$ is $\frac{1}{2 \eta}$-ism.

(4) If $T_{1}$ is $\eta_{1}$-averaged and $T_{2}$ is $\eta_{2}$-averaged, where $\eta_{1}, \eta_{2} \in(0,1)$, then $T_{1} o T_{2}$ is $\eta$-averaged, where $\eta=\eta_{1}+\eta_{2}-\eta_{1} \eta_{2}$

(5) If $T_{1}$ and $T_{2}$ are averaged and have a common fixed point, then $F\left(T_{1} o T_{2}\right)=F\left(T_{1}\right) \cap F\left(T_{2}\right)$.

Lemma 2.3. [18] Let $H_{1}$ and $H_{2}$ be real Hilbert spaces. Let $A: H_{1} \rightarrow H_{2}$ be a bounded linear operator with $A \neq 0$. Let $T: H_{2} \rightarrow H_{2}$ be a nonexpansive mapping. Then, $A^{*}(I-T) A$ is $\frac{1}{2\|A\|^{2}}$-ism.

Lemma 2.4. [19] Let $H_{1}$ and $H_{2}$ be real Hilbert spaces, and let $M$ be a nonempty closed and convex subset of $H_{1}$. Let $A: H_{1} \rightarrow H_{2}$ be a bounded linear operator. Let $T: H_{2} \rightarrow H_{2}$ be a nonexpansive mapping. Let $\gamma>0$ and $z \in H_{1}$. Suppose that $M \cap A^{-1} F(T) \neq \emptyset$. Then, the following are equivalent:

(1) $\left.z=P_{M}\left(I-\gamma A^{*}(I-T) A\right) z, \quad(2) 0 \in A^{*}(I-T) A\right) z+N_{M} z \quad$ and $\quad(3) z \in M \cap A^{-1} F(T)$.

Lemma 2.5. [12] Let $f: H \rightarrow H$ be a monotone and L-Lipschitz mapping on $M$. Let $U:=P_{M}(I-\tau f)$, where $\tau>0$. If $\left\{x_{n}\right\}$ is a sequence in $M$ such that $x_{n} \rightarrow x^{*}$ and $x_{n}-U\left(x_{n}\right) \rightarrow 0$, then $x^{*} \in \operatorname{VI}(M, f)=$ $F(U)$.

Definition 2.3. The generalized split feasibility problems $(G S F P)$ is to find an element $x^{*} \in M$ such that the following conditions hold:

$$
x^{*} \in V(C, f) \text { and } A x^{*} \in F(T),
$$

where $C$ is a nonempty closed and convex subset of real Hilbert space $H_{1}, f$ is mapping on $H_{1}, T$ is a mapping on $H_{2}$ and $B$ is a bounded linear mapping from $H_{1}$ to $H_{2}$.

Definition 2.4. Let $M$ be a nonempty closed convex subset of a real Hilbert space $H$, and let $\mathscr{G}: M \times M \rightarrow$ $\mathbb{R}$ be a mapping. The equilibrium problem is to find $x^{*} \in M$ such that

$$
\mathscr{G}\left(x^{*} \cdot y\right) \geq 0, \forall y \in M .
$$

In this paper, we denote the set of solutions of the equilibrium problem by $E P(\mathscr{G})$.

Remark 2.2. Let $M$ be a nonempty closed and convex subset of a real Hilbert space $H$. For solving the equilibrium problem, we assume that $\mathscr{G}: M \times M \rightarrow \mathbb{R}$ satisfies the following conditions:

$\left(P_{1}\right) \mathscr{G}(x, x)=0$, for all $x \in M$,

$\left(P_{2}\right) \mathscr{G}$ is monotone, i.e. $\mathscr{G}(x, y)+\mathscr{G}(y, x) \leq 0$, for all $x, y \in M$,

$\left(P_{3}\right) \limsup _{t \downarrow 0} \mathscr{G}(x+t(z-x), y) \leq \mathscr{G}(x, y)$, for all $x, y, z \in M$,

$\left(P_{4}\right)$ For all $x \in M, \mathscr{G}(x, \cdot)$ is convex and lower semi-continuous.

Definition 2.5. Let $M$ be a nonempty closed convex subset of a real Hilbert space $H$. The convex minimization problem is to find $x^{*} \in M$ such that

$$
\Theta\left(x^{*}\right)=\min _{x \in M} \Theta(x) .
$$


In this paper, we denote the set of solutions of the convex minimization problem by $\operatorname{Argmin}(M, \Theta)$.

Lemma 2.6. (Tiang and Jiang [19]) Let $M$ be a nonempty closed convex subset of a real Hilbert space $H$. Let $\Theta$ be a convex function of $H$ into $\mathbb{R}$. If $\Theta$ is differentiable, then $z$ is a solutions of constrained convex minimization problem (2.3) if and only if $z \in V I\left(M, \Theta^{\prime}\right)$.

\section{MAIN RESULTS}

\subsection{A hybrid method for the generalized split feasibility problem (GSFP).}

Theorem 3.1. Let $H_{1}$ and $H_{2}$ be real Hilbert spaces. Let $M_{i}, i=1, \cdots, N$ be nonempty closed and convex subsets of $H_{1}$ such that $M=\cap_{i=1}^{N} M_{i} \neq \emptyset$. Let $\nabla: H_{1} \rightarrow H_{2}$ be a bounded linear map such that $\nabla \neq 0$ and let $\nabla^{*}$ be the adjoint of $\nabla$. Let $B_{i}: M_{i} \rightarrow H_{1} i=1, \cdots, N$ be a finite family of monotone and L-Lipschitz mappings and let $T_{i}: H_{2} \rightarrow H_{2} i=1, \cdots, N$ be a finite family of nonexpansive mappings such that $\mathscr{D}=\left\{z \in \cap_{i=1}^{N} \operatorname{VI}\left(M_{i}, B_{i}\right): \nabla z \in \cap_{i=1}^{N} F\left(T_{i}\right)\right\} \neq \emptyset$. For $v_{1}=v \in H_{1}, C_{1}=H_{1}$, and $W_{1}=H_{1}$, let $\left\{v_{n}\right\}$ be a sequence given by:

$$
\left\{\begin{array}{l}
y_{n}^{i}=P_{M_{i}}\left(v_{n}-\lambda \nabla^{*}\left(I-T_{i}\right) \nabla v_{n}\right), i=1, \cdots, N, \\
u_{n}^{i}=P_{M_{i}}\left(y_{n}^{i}-\delta B_{i}\left(y_{n}^{i}\right)\right), i=1, \cdots, N, \\
t_{n}^{i}=P_{M_{i}}\left(y_{n}^{i}-\delta B_{i}\left(u_{n}^{i}\right)\right), i=1, \cdots, N, \\
C_{n}^{i}=\left\{z \in H:\left\|t_{n}^{i}-z\right\| \leq\left\|v_{n}-z\right\|\right\}, \\
W_{n}=\left\{z \in H:\left\langle z-v_{n}, v-v_{n}\right\rangle \leq 0\right\} \\
v_{n+1}=P_{C_{n} \cap W_{n}} v, \forall n \geq 1,
\end{array}\right.
$$

where $C_{n}=\cap_{i=1}^{N} C_{n}^{i}, \lambda \in\left(0, \frac{1}{\|\nabla\|^{2}}\right)$ and $\delta \in\left(0, \frac{1}{L}\right)$. The $\left\{v_{n}\right\}$ converges strongly to $P_{\mathscr{D}} v$.

Proof. Step 1. The sequence $\left\{v_{n}\right\}$ is well defined and $\mathscr{D} \subset C_{n} \cap W_{n}$.

First, we show that $C_{n}^{i}$ and $W_{n}$ are closed and convex. Clearly, from the definition of $C_{n}^{i}$ and $W_{n}$, they are either half-spaces or the whole space $H$. Thus, they are closed and convex. Therefore, $\left\{v_{n}\right\}$ is well defined.

Next, we prove that $\mathscr{D} \subset C_{n} \cap W_{n}$ for each $n \geq 1$.

Claim 1. $\mathscr{D} \subset C_{n}^{i}$ for each $n \geq 1$. Let $u \in \mathscr{D}$. Then, we compute as follows. From (3),(4) of Lemma 2.2, and Lemma 2.3, we see that $P_{M_{i}}\left(I-\lambda \nabla^{*}\left(I-T_{i}\right) \nabla\right)$ is $\frac{1+\lambda\|\nabla\|^{2}}{2}$ - averaged, for each $i=1, \cdots, N$. Hence, $y_{n}^{i}$ can be expressed as

$$
y_{n}^{i}=(1-\beta) v_{n}+\beta Q_{n}^{i} v_{n},
$$

where $\beta=\frac{1+\lambda\|\nabla\|^{2}}{2}$ and $Q_{n}^{i}$ is a nonxpansive mapping, for each $n \geq 1$ and for each $i=1, \cdots, N$.

$$
\begin{aligned}
\left\|y_{n}^{i}-u\right\|^{2} & =\left\|(1-\beta) v_{n}+\beta Q_{n}^{i} v_{n}-u\right\|^{2} \\
& =(1-\beta)\left\|v_{n}-u\right\|^{2}+\beta\left\|Q_{n}^{i} v_{n}-u\right\|^{2}-\beta(1-\beta)\left\|v_{n}-Q_{n}^{i} v_{n}\right\|^{2} \\
& \leq\left\|v_{n}-u\right\|^{2}-\beta(1-\beta)\left\|v_{n}-Q_{n}^{i} v_{n}\right\|^{2} .
\end{aligned}
$$

Since $u_{n}^{i} \in M_{i}$ and $B_{i}$ is monotone, for each $i=1, \cdots, N$, we have that

$$
\left\langle u_{n}^{i}-u, B_{i}\left(u_{n}^{i}\right)-B_{i}(u)\right\rangle \geq 0, \forall n \geq 1, i=1, \cdots, N .
$$

Since

$$
t_{n}^{i}=P_{M_{i}}\left(y_{n}^{i}-\delta B_{i}\left(u_{n}^{i}\right)\right)
$$


we have

$$
\left\langle u-t_{n}^{i}, B_{i}\left(u_{n}^{i}\right)\right\rangle \leq\left\langle u_{n}^{i}-t_{n}^{i}, B_{i}\left(u_{n}^{i}\right)\right\rangle .
$$

Set $z_{n}^{i}=y_{n}^{i}-\delta B_{i}\left(u_{n}^{i}\right)$. For each $i=1, \cdots, N$, we obtain from Lemma 2.1(1) that

$$
\begin{aligned}
\left\|t_{n}^{i}-u\right\|^{2} & \leq\left\|z_{n}^{i}-u\right\|^{2}-\left\|z_{n}^{i}-t_{n}^{i}\right\|^{2} \\
& =\left\|\left(y_{n}^{i}-u\right)-\delta B_{i}\left(u_{n}\right)\right\|^{2}-\left\|\left(y_{n}^{i}-t_{n}^{i}\right)-\delta B_{i}\left(u_{n}^{i}\right)\right\|^{2} \\
& =\left\|y_{n}^{i}-u\right\|^{2}-\left\|t_{n}^{i}-y_{n}^{i}\right\|^{2}+2 \delta\left\langle u-t_{n}^{i}, B_{i}\left(u_{n}^{i}\right)\right\rangle .
\end{aligned}
$$

From inequality (3.4), one has

$$
\begin{aligned}
\left\|t_{n}^{i}-u\right\|^{2} \leq & \left\|y_{n}^{i}-u\right\|^{2}-\left\|t_{n}^{i}-y_{n}^{i}\right\|^{2}+2 \delta\left\langle u_{n}^{i}-t_{n}^{i}, B_{i}\left(u_{n}^{i}\right)\right\rangle \\
= & \left\|y_{n}^{i}-u\right\|^{2}-\left\|y_{n}^{i}-u_{n}^{i}\right\|^{2}-\left\|u_{n}^{i}-t_{n}^{i}\right\|^{2}+2\left\langle t_{n}^{i}-u_{n}^{i}, y_{n}^{i}-\delta B_{i}\left(u_{n}^{i}\right)-u_{n}^{i}\right\rangle \\
= & \left\|y_{n}^{i}-u\right\|^{2}-\left\|y_{n}^{i}-u_{n}^{i}\right\|^{2}-\left\|u_{n}^{i}-t_{n}^{i}\right\|^{2}+2\left\langle t_{n}^{i}-u_{n}^{i}, y_{n}^{i}-\delta B_{i}\left(y_{n}^{i}\right)-u_{n}^{i}\right\rangle \\
& +2 \delta\left\langle t_{n}^{i}-u_{n}^{i}, B_{i}\left(y_{n}^{i}\right)-B_{i}\left(u_{n}^{i}\right)\right\rangle \\
\leq & \left\|y_{n}^{i}-u\right\|^{2}-\left\|y_{n}^{i}-u_{n}^{i}\right\|^{2}-\left\|u_{n}^{i}-t_{n}^{i}\right\|^{2}+2 \delta\left\langle t_{n}^{i}-u_{n}^{i}, B_{i}\left(y_{n}^{i}\right)-B_{i}\left(u_{n}^{i}\right)\right\rangle .
\end{aligned}
$$

Since $B_{i}$ is $L$-Lipschitz, for each $i=1, \cdots, N$, we see from inequality (3.6) that

$$
\begin{aligned}
\left\|t_{n}^{i}-u\right\|^{2} & \leq\left\|y_{n}^{i}-u\right\|^{2}-\left\|y_{n}^{i}-u_{n}^{i}\right\|^{2}-\left\|u_{n}^{i}-t_{n}^{i}\right\|^{2}+2 L \delta\left\|t_{n}^{i}-u_{n}^{i}\right\|\left\|y_{n}^{i}-u_{n}^{i}\right\| \\
& \leq\left\|y_{n}^{i}-u\right\|^{2}-\left\|y_{n}^{i}-u_{n}^{i}\right\|^{2}-\left\|u_{n}^{i}-t_{n}^{i}\right\|^{2}+L \delta\left(\left\|t_{n}^{i}-u_{n}^{i}\right\|^{2}+\left\|y_{n}^{i}-u_{n}^{i}\right\|^{2}\right) \\
& =\left\|y_{n}^{i}-u\right\|^{2}-(1-L \delta)\left\|y_{n}^{i}-u_{n}^{i}\right\|^{2}-(1-L \delta)\left\|u_{n}^{i}-t_{n}^{i}\right\|^{2} .
\end{aligned}
$$

From inequality (3.3), we obtain

$$
\left\|t_{n}^{i}-u\right\|^{2} \leq\left\|v_{n}-u\right\|^{2}-(1-L \delta)\left\|y_{n}^{i}-u_{n}^{i}\right\|^{2}-(1-L \delta)\left\|u_{n}^{i}-t_{n}^{i}\right\|^{2} \leq\left\|v_{n}-u\right\|^{2} .
$$

This implies that $\mathscr{D} \subset C_{n}^{i}$ for all $n \geq 1$ and for all $i=1, \cdots, N$. Hence, $\mathscr{D} \subset C_{n}=\cap_{i=1}^{N} C_{n}^{i}$.

Claim 2. $\mathscr{D} \subset C_{n} \cap W_{n}$ for all $n \in \mathbb{N}$. Clearly, $\mathscr{D} \subset C_{1} \cap W_{1}$. Assume that $\mathscr{D} \subset C_{n} \cap W_{n}$ for some $n \geq 1$. From $v_{n+1}=P_{C_{n} \cap W_{n}} v$ and Lemma 2.1(2), we have that $\left\langle z-v_{n+1}, v-v_{n+1}\right\rangle \leq 0$, for all $z \in C_{n} \cap W_{n}$. In particular, $u \in \mathscr{D} \subset C_{n} \cap W_{n}$. Using the definition of $W_{n+1}, \mathscr{D} \subset W_{n+1}$, which implies $\mathscr{D} \subset C_{n+1} \cap W_{n+1}$. Hence, $\mathscr{D} \subset C_{n} \cap W_{n}$ for each $n \geq 1$.

Step 2.

$$
\lim _{n \rightarrow \infty}\left\|v_{n}-t_{n}^{i}\right\|=\lim _{n \rightarrow \infty}\left\|y_{n}^{i}-u_{n}^{i}\right\|=\lim _{n \rightarrow \infty}|| u_{n}^{i}-t_{n}^{i}\left\|=\lim _{n \rightarrow \infty}\right\| u_{n}^{i}-v_{n} \|=0, \quad \forall i=1, \cdots, N .
$$

First, we prove that $\left\{v_{n}\right\}$ is bounded. From the definition of $\left\{W_{n}\right\}$, we have that $v_{n}=P_{W_{n}} v, \forall n \geq 1$. Hence, by Lemma 2.1 (1), we obtain that

$$
\left\|v_{n}-v\right\|^{2}=\left\|P_{W_{n}} v-v\right\|^{2} \leq\|u-v\|^{2}-\left\|u-v_{n}\right\|^{2} \leq\|u-v\|^{2}, \forall u \mathscr{D} \subset W_{n} .
$$

This implies that $\left\{\left\|v_{n}-v\right\|\right\}$ is bounded. Hence, $\left\{v_{n}\right\}$ is bounded. Consequently, $\left\{t_{n}^{i}\right\},\left\{y_{n}^{i}\right\}$, and $\left\{u_{n}^{i}\right\}$ are bounded for each $i=, 1 \cdots, N$. Since $v_{n+1}=P_{C_{n} \cap W_{n}} v \in W_{n}$ and $v_{n}=P_{W_{n}} v$, we conclude from the definition of $v_{n}=P_{W_{n}} v$ that $\left\|v_{n}-v\right\| \leq\left\|v_{n+1}-v\right\|, \forall n \geq 1$. This implies that $\left\{\left\|v_{n}-v\right\|\right\}$ is monotone nondecreasing. Hence, $\lim _{n \rightarrow \infty}\left\|v_{n}-v\right\|$ exists. 
From Lemma 2.1(1) and $v_{n}=P_{W_{n}} v$, we obtain, for arbitrary $m, n \in \mathbb{N}$, with $m>n$, that

$$
\begin{aligned}
\left\|v_{m}-v_{n}\right\|^{2}=\left\|v_{m}-P_{W_{n}} v\right\|^{2} & \leq\left\|v_{m}-v\right\|^{2}-\left\|P_{W_{n}} v-v\right\|^{2} \\
& =\left\|v_{m}-v\right\|^{2}-\left\|v_{n}-v\right\|^{2} \rightarrow 0(\text { as } n, m \rightarrow \infty) .
\end{aligned}
$$

Hence, $\left\{v_{n}\right\}$ is Cauchy. Thus, there exists $x^{*} \in M$ such that $\lim _{n \rightarrow \infty} v_{n}=x^{*}$. Since $v_{m} \in C_{m} \subset C_{n}=\cap_{i=1}^{N} C_{n}^{i}$, we obtain from the definition $C_{n}^{i}$ that $\left\|t_{n}^{i}-v_{m}\right\| \leq\left\|v_{n}-v_{m}\right\|$. Using this and inequality (3.10), we have that

$$
\left\|v_{n}-t_{n}^{i}\right\| \leq\left\|v_{n}-v_{m}\right\|+\left\|v_{m}-t_{n}^{i}\right\| \leq 2\left\|v_{n}-v_{m}\right\| \rightarrow 0(\text { for each } i=1, \cdots, N) .
$$

From inequality (3.8), we set $\gamma=(1-L \delta)^{-1}$ such that

$$
\begin{aligned}
\left\|y_{n}^{i}-u_{n}^{i}\right\|^{2} & \leq \gamma\left(\left\|v_{n}-u\right\|^{2}-\left\|t_{n}^{i}-u\right\|^{2}\right) \leq \gamma\left(\left\|v_{n}-u\right\|+\left\|t_{n}^{i}-u\right\|\right)\left(\left\|v_{n}-t_{n}^{i}\right\|\right), \text { and } \\
\left\|u_{n}^{i}-t_{n}^{i}\right\|^{2} & \leq \gamma\left(\left\|v_{n}-u\right\|^{2}-\left\|t_{n}^{i}-u\right\|^{2}\right) \leq \gamma\left(\left\|v_{n}-u\right\|+\left\|t_{n}^{i}-u\right\|\right)\left(\left\|v_{n}-t_{n}^{i}\right\|\right) .
\end{aligned}
$$

From inequality (3.11), we obtain that

$$
\lim _{n \rightarrow \infty}\left\|y_{n}^{i}-u_{n}^{i}\right\|=\lim _{n \rightarrow \infty}\left\|u_{n}^{i}-t_{n}^{i}\right\|=0, \quad \text { for each } i=1, \cdots, N
$$

From (3.3) and (3.7), we obtain that

$$
\beta(1-\beta)\left\|v_{n}-Q_{n}^{i} v_{n}\right\|^{2} \leq\left\|v_{n}-u\right\|^{2}-\left\|y_{n}^{i}-u\right\|^{2} \leq\left\|v_{n}-u\right\|^{2}-\left\|t_{n}^{i}-u\right\|^{2} .
$$

Hence, we conclude from inequality (3.11) that, for each $i=1, \cdots, N, \lim _{n \rightarrow \infty}\left\|v_{n}-Q_{n}^{i} v_{n}\right\|=0$. We obtain from equation (3.2) that $\lim _{n \rightarrow \infty}\left\|y_{n}^{i}-v_{n}\right\|=0$. Hence,

$$
\lim _{n \rightarrow \infty}\left\|v_{n}-t_{n}^{i}\right\|=\lim _{n \rightarrow \infty}\left|\left\|y_{n}^{i}-u_{n}^{i}\right\|=\lim _{n \rightarrow \infty}\right| \mid u_{n}^{i}-t_{n}^{i}\left\|=\lim _{n \rightarrow \infty}\right\| u_{n}^{i}-v_{n} \|=0, \quad \text { for each } i=1, \cdots, N .
$$

Step 3. $\Omega_{\omega}\left(v_{n}\right) \subset \cap_{i=1}^{N}\left(M_{i} \cap B_{i}^{-1} F\left(T_{i}\right)\right)$ and $\Omega_{\omega}\left(v_{n}\right) \subset \cap_{i=1}^{N} V I\left(M_{i}, B_{i}\right)$, where $\Omega_{\omega}\left(v_{k}\right)$ is the set of weak sub-sequential limits of $\left\{v_{n}\right\}$.

Let $x^{*} \in \Omega_{\omega}\left(v_{n}\right)$ and $\left\{v_{n_{j}}\right\}_{j=1}^{\infty}$ be a subsequence of $\left\{v_{n}\right\}_{n=1}^{\infty}$ such that

$$
v_{n_{j}} \rightarrow x^{*} \text { as } j \rightarrow \infty \text {. Consequently, } u_{n_{j}}^{i} \rightarrow x^{*} \text { as } j \rightarrow \infty \text {, for each } i=1, \cdots, N .
$$

From the definition of $y_{n}^{i}$, we have that

$$
\lim _{j \rightarrow \infty}\left\|v_{n_{j}}-P_{M_{i}}\left(v_{n_{j}}-\lambda \nabla^{*}\left(I-T_{i}\right) \nabla v_{n_{j}}\right)\right\|=0,
$$

for each $i=1, \cdots, N$. Since, for each $i=1, \cdots, N, \nabla^{*}\left(I-T_{i}\right) \nabla$ is ism, then it is Lipschitz. By Lemma 2.5, we obtain that $x^{*} \in F\left(P_{M_{i}}\left(I-\lambda \nabla^{*}\left(I-T_{i}\right) \nabla\right)\right)$, for each $i=1, \cdots, N$. By Lemma 2.4, we have that $\Omega_{\omega}\left(v_{n}\right) \subset M_{i} \cap B^{-1} F\left(T_{i}\right)$, for each $i=1, \cdots, N$.

Next, we show that $\Omega_{\omega}\left(v_{n}\right) \subset \cap_{i=1}^{N} V I\left(M_{i}, B_{i}\right)$. Let $x^{*} \in \Omega_{\omega}\left(v_{n}\right)$ and $\left\{u_{n_{j}}^{i}\right\}_{j=1}^{\infty}$ be a subsequence of $\left\{u_{n}^{i}\right\}_{n=1}^{\infty}$ such that $u_{n_{j}}^{i} \rightarrow x^{*}$ as $j \rightarrow \infty$, for each $i=1, \cdots, N$. From a result of [16, Rockafellar], we define mappings $R_{i}: H \rightarrow H i=1, \cdots, N$ by

$$
R_{i} u= \begin{cases}B_{i} u+N_{M_{i}}(u), & \text { if } u \in M_{i}, \\ \emptyset, & \text { if } u \notin M_{i},\end{cases}
$$


where $N_{M_{i}}(\cdot)$ is the normal cone of $M_{i}$, for each $i=1, \cdots, N$. Then, $R_{i}$ is maximal monotone and $R_{i}^{-1}(0)=V I\left(M_{i}, B_{i}\right)$, for each $i=1, \cdots, N$. Let $(u, w) \in G\left(R_{i}\right)$, where $G\left(R_{i}\right)$ is the graph of $R_{i}$, for each $i=1, \cdots, N$. Then,

$$
w \in R_{i} u=B_{i} u+N_{M_{i}}(u) .
$$

Hence, we get that $w-B_{i} u \in N_{M_{i}}(u)$. This implies that $\left\langle u-t, w-B_{i} u\right\rangle \geq 0, \forall t \in M_{i}$. In particular,

$$
\left\langle u-u_{n}^{i}, w-B_{i} u\right\rangle \geq 0 .
$$

But $u_{n}^{i}=P_{M_{i}}\left(y_{n}^{i}-\delta B_{i}\left(y_{n}^{i}\right)\right), \forall n \geq 1$ and for each $i=1, \cdots, N$. By a characterization of the metric projection, we obtain that

$$
\left\langle u_{n}^{i}-u, y_{n}^{i}-\delta B_{i}\left(y_{n}^{i}\right)-u_{n}\right\rangle \geq 0, \forall u \in M_{i}
$$

This implies that

$$
\left\langle u-u_{n}^{i}, \frac{u_{n}^{i}-y_{n}^{i}}{\delta}+B_{i}\left(y_{n}^{i}\right)\right\rangle \geq 0, \forall u \in M_{i}
$$

Using inequalities (3.13) and (3.15) for some $M_{0}>0$, and the fact that $B_{i}$ is monotone and $L$-Lipschitz, for each $i=1, \cdots, N$, we have that

$$
\begin{aligned}
\left\langle u-u_{n_{j}}^{i}, w\right\rangle & \geq\left\langle u-u_{n_{j}^{i}}, B_{i} u\right\rangle \\
& \geq\left\langle u-u_{n_{j}}^{i}, B_{i} u\right\rangle-\left\langle u-u_{n_{j}}^{i}, \frac{u_{n_{j}}^{i}-y_{n_{j}}^{i}}{\delta}+B_{i}\left(y_{n_{j}}^{i}\right)\right\rangle \\
& =\left\langle u-u_{n_{j}}^{i}, B_{i} u-B_{i}\left(u_{n_{j}}^{i}\right)\right\rangle+\left\langle u-u_{n_{j}}^{i}, B\left(u_{n_{j}}^{i}\right)-B_{i}\left(y_{n_{j}}^{i}\right)\right\rangle-\left\langle u-u_{n_{j}}^{i}, \frac{u_{n_{j}}^{i}-y_{n_{j}}^{i}}{\delta}\right\rangle \\
& \geq-M_{0}(1+L)\left\|u_{n_{j}}^{i}-y_{n_{j}}^{i}\right\| .
\end{aligned}
$$

Taking limit of both sides of inequality (3.16) as $j \rightarrow \infty$, for each $i=1, \cdots, N$, we obtain that

$$
\left\langle u-x^{*}, w\right\rangle \geq 0 \text {. }
$$

Since $R_{i}$ is maximal monotone for each $i=1, \cdots, N$, it follows that $x^{*} \in R_{i}^{-1}(0)=\operatorname{VI}\left(M_{i}, B_{i}\right)$, which implies that $\Omega_{\omega}\left(v_{n}\right) \subset V I\left(M_{i}, B_{i}\right)$, for each $i=1, \cdots, N$. Hence, $\Omega_{\omega}\left(v_{n}\right) \subset \mathscr{D}$.

Step 4. $\lim _{n \rightarrow \infty} v_{n}=P_{\mathscr{D}} v$.

From Lemma 2.1(1), we have that

$$
\left\|v-P_{\mathscr{D}} v\right\| \leq\left\|v-x^{*}\right\| .
$$

For $x^{*} \in \mathscr{D} \subset W_{n}, v_{n}=P_{W_{n}}$ and Lemma 2.1(1), we obtain that $\left\|v-v_{n}\right\| \leq\left\|v-P_{\mathscr{D}}\right\|$. Since, $\lim _{n \rightarrow \infty} v_{n}=x^{*}$,

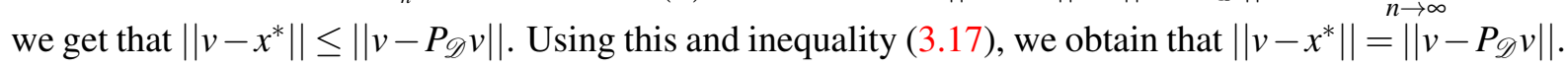
By uniqueness of $P_{\mathscr{D}} v$, we conclude that $x^{*}=P_{\mathscr{D}} v$. This proof is complete.

\subsection{A hybrid method for the common split variational inequality problem (CSSVIP).}

Theorem 3.2. Let $H_{1}$ and $H_{2}$ be real Hilbert spaces. Let $M$ and $Q$ be nonempty closed convex subsets of $H_{1}$ and $H_{2}$, respectively, such that $M=\cap_{i=1}^{N} M_{i} \neq \emptyset$ and $Q=\cap_{i=1}^{N} Q_{i} \neq \emptyset$. Let $\nabla: H_{1} \rightarrow H_{2}$ be a bounded linear map such that $\nabla \neq 0$, and $\nabla^{*}$ be the adjoint of $\nabla$. Let $B_{i}: M_{i} \rightarrow H_{1} i=1, \cdots, N$, be monotone and L-Lipschitz maps. Let $\mathscr{F}_{i}: H_{2} \rightarrow H_{2} i=1, \cdots, N$, be $\eta$-inverse strongly monotone map such that 
$\mathscr{D}=\left\{z \in \cap_{i=1}^{N} \operatorname{VI}\left(M_{i}, B_{i}\right): \nabla z \in \cap_{i=1}^{N} V I\left(Q_{i}, \mathscr{F}_{i}\right)\right\} \neq \emptyset$. For $v_{1}=v \in H_{1}, C_{1}=H_{1}$, and $W_{1}=H_{1}$, with $T_{i}=P_{Q_{i}}\left(I-\mu \mathscr{F}_{i}\right)$ and $\mu \in(0,2 \eta)$. Then $\left\{v_{n}\right\}$ generated by equation (3.1) converges strongly to $P_{\mathscr{D} V}$.

Proof. From Lemma 2.1, it is easy to see that $u \in V I\left(Q_{i}, \mathscr{F}_{i}\right)$ if and only if $u=P_{Q_{i}}\left(I-\mu \mathscr{F}_{i}\right) u$, for $\mu>0$. Furthermore, $P_{Q_{i}}\left(I-\mu \mathscr{F}_{i}\right)$ is nonexpansive if $\mu \in(0,2 \eta)$. Hence, by Theorem 3.1, the result of Theorem 3.2 is immediate.

\subsection{A modified hybrid method for the generalized split feasibility problem.}

Theorem 3.3. Let $H_{1}$ and $H_{2}$ be real Hilbert spaces. Let $M_{i}, i=1, \cdots, N$ be nonempty closed and convex subsets of $H_{1}$ such that $M=\cap_{i=1}^{N} M_{i} \neq \emptyset$. Let $\nabla: H_{1} \rightarrow H_{2}$ be a bounded linear map such that $\nabla \neq 0$ and $\nabla^{*}$ be the adjoint of $\nabla$. Let $B_{i}: M_{i} \rightarrow H_{1} i=1, \cdots, N$ be a finite family of monotone and L-Lipschitz maps and $T_{i}: H_{2} \rightarrow H_{2} i=1, \cdots, N$ be a finite family of nonexpansive maps such that $\mathscr{D}=\left\{z \in \cap_{i=1}^{N} V I\left(M_{i}, B_{i}\right): \nabla z \in \cap_{i=1}^{N} F\left(T_{i}\right)\right\} \neq \emptyset$. For $v_{1}=v \in H_{1}, C_{1}^{i}=H_{1}$, let $\left\{v_{n}\right\}$ be a sequence given by:

$$
\left\{\begin{array}{l}
y_{n}^{i}=P_{M_{i}}\left(v_{n}-\lambda \nabla^{*}\left(I-T_{i}\right) \nabla v_{n}\right), i=1, \cdots, N, \\
u_{n}^{i}=P_{M_{i}}\left(y_{n}^{i}-\delta B_{i}\left(y_{n}^{i}\right)\right), i=1, \cdots, N, \\
t_{n}^{i}=P_{M_{i}}\left(y_{n}^{i}-\delta B_{i}\left(u_{n}^{i}\right)\right), i=1, \cdots, N, \\
C_{n+1}^{i}=\left\{z \in C_{n}^{i}:\left\|t_{n}^{i}-z\right\| \leq\left\|v_{n}-z\right\|\right\}, \\
v_{n+1}=P_{C_{n+1}} v, \forall n \geq 1,
\end{array}\right.
$$

where $C_{n+1}=\cap_{i=1}^{N} C_{n+1}^{i}, \lambda \in\left(0, \frac{1}{\|\nabla\|^{2}}\right)$ and $\delta \in\left(0, \frac{1}{L}\right)$. Then, $\left\{v_{n}\right\}$ converges strongly to $P_{\mathscr{D} V}$.

Proof. Step 1. Show that $\left\{v_{n}\right\}$ is well defined and $\mathscr{D} \subset C_{n}$.

By induction, we can easily show that $C_{n}$ is the intersection of finitely many closed half spaces which are also convex. Therefore, $\left\{v_{n}\right\}$ is well defined. Next, we prove that $\mathscr{D} \subset C_{n}$ for each $n \geq 1$.

Claim 1. $\mathscr{D} \subset C_{n}^{i}$ for each $n \geq 1$. Clearly, $\mathscr{D} \subset C_{1}^{i}=H_{1}$. Assume that $\mathscr{D} \subset C_{n}^{i}$ for some $n \geq 1$. Let $u \in \mathscr{D}$. By a similar argument as in Claim 1 of Theorem 3.1, we obtain that

$$
\left\|t_{n}^{i}-u\right\|^{2} \leq\left\|v_{n}-u\right\|^{2}-(1-L \delta)\left\|y_{n}^{i}-u_{n}^{i}\right\|^{2}-(1-L \delta)\left\|u_{n}^{i}-t_{n}^{i}\right\|^{2} \leq\left\|v_{n}-u\right\|^{2},
$$

which implies that $\mathscr{D} \subset C_{n+1}^{i}$. Hence, $\mathscr{D} \subset C_{n+1}=\cap_{i=1}^{N} C_{n+1}^{i}, \forall n \geq 1$.

Step 2. $\lim _{n \rightarrow \infty}\left\|v_{n}-t_{n}^{i}\right\|=\lim _{n \rightarrow \infty}|| y_{n}^{i}-u_{n}^{i}\left\|=\lim _{n \rightarrow \infty}|| u_{n}^{i}-t_{n}^{i}\right\|=\lim _{n \rightarrow \infty}\left\|u_{n}^{i}-v_{n}\right\|=0$, for each $i=1, \cdots, N$.

First, we prove that $\left\{v_{n}\right\}$ is bounded. From the definition, we have that $v_{n}=P_{C_{n}} v, \forall n \geq 1$. Hence, by Lemma 2.1(1), we obtain that

$$
\left\|v_{n}-v\right\|^{2}=\left\|P_{C_{n}} v-v\right\|^{2} \leq\|u-v\|^{2}-\left\|u-v_{n}\right\|^{2} \leq\|u-v\|^{2}, \forall u \mathscr{D} \subset C_{n} .
$$

This implies that $\left\{\left\|v_{n}-v\right\|\right\}$ is bounded. Hence, $\left\{v_{n}\right\}$ is bounded. Consequently, $\left\{t_{n}^{i}\right\},\left\{y_{n}^{i}\right\}$, and $\left\{u_{n}^{i}\right\}$ are bounded for each $i=, 1 \cdots, N$. Since $v_{n+1}=P_{C_{n+1}} v \in C_{n}$ and $v_{n}=P_{C_{n}} v$, we have that

$$
\left\|v_{n}-v\right\| \leq\left\|v_{n+1}-v\right\|, \quad \forall n \geq 1,
$$

which implies that $\left\{\left\|v_{n}-v\right\|\right\}$ is monotone nondecreasing. Hence, $\lim _{n \rightarrow \infty}\left\|v_{n}-v\right\|$ exists. From Lemma 2.1(1) and $v_{n}=P_{C_{n}} v$, we obtain, for arbitrary $m, n \in \mathbb{N}$, with $m>n$, that

$$
\begin{aligned}
\left\|v_{m}-v_{n}\right\|^{2}=\left\|v_{m}-P_{C_{n}} v\right\|^{2} & \leq\left\|v_{m}-v\right\|^{2}-\left\|P_{C_{n}} v-v\right\|^{2} \\
& =\left\|v_{m}-v\right\|^{2}-\left\|v_{n}-v\right\|^{2} \rightarrow 0(\text { as } n, m \rightarrow \infty) .
\end{aligned}
$$


Hence, $\left\{v_{n}\right\}$ is Cauchy. Thus, there exists $x^{*} \in M$ such that $\lim _{n \rightarrow \infty} v_{n}=x^{*}$. Since $v_{m} \in C_{m} \subset C_{n}=\cap_{i=1}^{N} C_{n}^{i}$, we obtain from the definition $C_{n}^{i}$ that ||$t_{n}^{i}-v_{m}\|\leq\| v_{n}-v_{m} \|$. Using this and inequality (3.21), we have that

$$
\left\|v_{n}-t_{n}^{i}\right\| \leq\left\|v_{n}-v_{m}\right\|+\left\|v_{m}-t_{n}^{i}\right\| \leq 2\left\|v_{n}-v_{m}\right\| \rightarrow 0(\text { for each } i=1, \cdots, N) .
$$

From inequality (3.19), we set $\gamma=(1-L \delta)^{-1}$ such that

$$
\begin{aligned}
\left\|y_{n}^{i}-u_{n}^{i}\right\|^{2} & \leq \gamma\left(\left\|v_{n}-u\right\|^{2}-\left\|t_{n}^{i}-u\right\|^{2}\right) \leq \gamma\left(\left\|v_{n}-u\right\|+\left\|t_{n}^{i}-u\right\|\right)\left(\left\|v_{n}-t_{n}^{i}\right\|\right), \text { and } \\
\left\|u_{n}^{i}-t_{n}^{i}\right\|^{2} & \leq \gamma\left(\left\|v_{n}-u\right\|^{2}-\left\|t_{n}^{i}-u\right\|^{2}\right) \leq \gamma\left(\left\|v_{n}-u\right\|+\left\|t_{n}^{i}-u\right\|\right)\left(\left\|v_{n}-t_{n}^{i}\right\|\right) .
\end{aligned}
$$

From inequality (3.22), we obtain that

$$
\lim _{n \rightarrow \infty}\left\|y_{n}^{i}-u_{n}^{i}\right\|=\lim _{n \rightarrow \infty}\left\|u_{n}^{i}-t_{n}^{i}\right\|=0, \quad \text { for each } i=1, \cdots, N .
$$

Furthermore, from inequality (3.3), as in the proof Theorem 3.1, we can obtain that

$$
\beta(1-\beta)\left\|v_{n}-Q_{n}^{i} v_{n}\right\|^{2} \leq\left\|v_{n}-u\right\|^{2}-\left\|y_{n}^{i}-u\right\|^{2} \leq\left\|v_{n}-u\right\|^{2}-\left\|t_{n}^{i}-u\right\|^{2} .
$$

Hence, from inequality (3.22), we conclude that, for each $i=1, \cdots, N, \lim _{n \rightarrow \infty}\left\|v_{n}-Q_{n}^{i} v_{n}\right\|=0$. From equation (3.2), as in the proof of Theorem 3.1, we obtain that $\lim _{n \rightarrow \infty}|| y_{n}^{i}-v_{n} \|=0$. Hence,

$$
\lim _{n \rightarrow \infty}\left\|v_{n}-t_{n}^{i}\right\|=\lim _{n \rightarrow \infty}|| y_{n}^{i}-u_{n}^{i}\left\|=\lim _{n \rightarrow \infty}\right\| u_{n}^{i}-t_{n}^{i}\left\|=\lim _{n \rightarrow \infty}\right\| u_{n}^{i}-v_{n} \|=0, \quad \text { for each } i=1, \cdots, N .
$$

Step 3. The rest of the proof of Theorem 3.3 is similar to Theorem 3.1. This completes the proof.

\subsection{A modified hybrid method for the common split variational inequality problem (CSSVIP).}

Theorem 3.4. Let $H_{1}$ and $H_{2}$ be real Hilbert spaces. Let $M$ and $Q$ be nonempty closed convex subsets of $H_{1}$ and $H_{2}$, respectively such that $M=\cap_{i=1}^{N} M_{i} \neq \emptyset$ and $Q=\cap_{i=1}^{N} Q_{i} \neq \emptyset$. Let $\nabla: H_{1} \rightarrow H_{2}$ be a bounded linear mapping such that $\nabla \neq 0$, and let $\nabla^{*}$ be the adjoint of $\nabla$. Let $B_{i}: M_{i} \rightarrow H_{1} i=1, \cdots, N$ be monotone and L-Lipschitz mappings. Let $\mathscr{F}_{i}: H_{2} \rightarrow H_{2} i=1, \cdots, N$ be $\eta$-inverse strongly monotone mapping such that $\mathscr{D}=\left\{z \in \cap_{i=1}^{N} V I\left(M_{i}, B_{i}\right): \nabla z \in \cap_{i=1}^{N} V I\left(Q_{i}, \mathscr{F}_{i}\right)\right\} \neq \emptyset$. For $v_{1}=v \in H_{1}, C_{1}^{i}=H_{1}$ with $T_{i}=P_{Q_{i}}\left(I-\mu \mathscr{F}_{i}\right)$ and $\mu \in(0,2 \eta)$, let $\left\{v_{n}\right\}$ be a sequence generated in (3.18). Then $\left\{v_{n}\right\}$ converges strongly to $P_{\mathscr{D} V}$.

Proof. From Lemma 2.1, it is easy to see that $u \in V I\left(Q_{i}, \mathscr{F}_{i}\right)$ if and only if $u=P_{Q_{i}}\left(I-\mu \mathscr{F}_{i}\right) u$, for $\mu>0$. Furthermore, $P_{Q_{i}}\left(I-\mu \mathscr{F}_{i}\right)$ is nonexpansive if $\mu \in(0,2 \eta)$. From Theorem 3.3, one can obtain the desired conclusion immediately.

\section{APPLICATIONS}

In this section, we apply our theorem to solve equilibrium problems and optimization problems.

Theorem 4.1. Let $H_{1}$ and $H_{2}$ be real Hilbert spaces. Let $M$ and $Q$ be nonempty closed and convex subsets of $H_{1}$ and $H_{2}$, respectively such that $M=\cap_{i=1}^{N} M_{i} \neq \emptyset$ and $Q=\cap_{i=1}^{N} Q_{i} \neq \emptyset$. Let $\nabla: H_{1} \rightarrow H_{2}$ be a bounded linear mapping such that $\nabla \neq 0$, and let $\nabla^{*}$ be the adjoint of $\nabla$. Let $B_{i}: M_{i} \rightarrow H_{1} i=1, \cdots, N$ be monotone and L-Lipschitz maps and let $\mathscr{G}_{i}: Q_{i} \times Q_{i} \rightarrow \mathbb{R} i=1, \cdots, N$, be bifunctions satisfying conditions $\left(P_{1}\right)$ $\left(P_{4}\right)$ such that $\mathscr{D}=\left\{z \in \cap_{i=1}^{N} V I\left(M_{i}, B_{i}\right): \nabla z \in \cap_{i=1}^{N} E P\left(\mathscr{G}_{i}\right)\right\} \neq \emptyset$. For $v_{1}=v \in H_{1}, C_{1}=H_{1}, W_{1}=H_{1}$, let $\left\{v_{n}\right\}$ be a sequence generated in equation (3.1). Then $\left\{v_{n}\right\}$ converges strongly to $P_{\mathscr{D}} v$. 
Proof. Set $T_{i}=T_{r}^{\mathscr{G}_{i}}$ for each $i=1, \cdots, N$, in Theorem 3.1. By Remark 2.2, results of Blum and Oettli [1], Combettes and Hirstoga [8], Theorem 4.1 is immediate.

Theorem 4.2. Let $H_{1}$ and $H_{2}$ be real Hilbert spaces. Let $M$ and $Q$ be nonempty closed and convex subsets of $H_{1}$ and $H_{2}$, respectively such that $M=\cap_{i=1}^{N} M_{i} \neq \emptyset$ and $Q=\cap_{i=1}^{N} Q_{i} \neq \emptyset$. Let $\nabla: H_{1} \rightarrow H_{2}$ be a bounded linear mapping such that $\nabla \neq 0$, and let $\nabla^{*}$ be the adjoint of $\nabla$. Let $B_{i}: M_{i} \rightarrow H_{1} i=1, \cdots, N$ be monotone and L-Lipschitz maps and let $\Theta_{i}: H_{2} \rightarrow \mathbb{R} i=1, \cdots, N$, be differentiable convex functions. Suppose that $\Theta_{i}^{\prime}$ is $\eta$-inverse strongly monotone such that $\mathscr{D}=\left\{z \in \cap_{i=1}^{N} V I\left(M_{i}, B_{i}\right): \nabla z \in \cap_{i=1}^{N} \operatorname{Argmin}\left(Q_{i}, \Theta_{i}\right)\right\} \neq \emptyset$. For $v_{1}=v \in H_{1}, C_{1}=H_{1}, W_{1}=H_{1}$, let $\left\{v_{n}\right\}$ be a sequence generated in equation (3.1). Then $\left\{v_{n}\right\}$ converges strongly to $P_{\mathscr{D}} v$.

Proof. Set $T_{i}=P_{Q_{i}}\left(I-\mu \Theta_{i}^{\prime}\right)$, for each $i=1, \cdots, N$, in Theorem 3.1 with $\mathscr{F}_{i}=\Theta_{i}^{\prime}$, for each $i=1, \cdots, N$, in Theorem 3.2. Using Lemma 2.6, Theorem 4.2 is immediate.

\section{NUMERICAL EXPERIMENTS}

In this section, we present numerical examples to illustrate the convergence of our sequence $\left\{v_{n}\right\}$ in Theorem 3.1 and Theorem 3.2, respectively. Set $N=1$.

Example 5.1. Let $H=\mathbb{R}, M=[-20,10], v_{1}=30, \nabla v=5 v, B v=10 v, \mathscr{F} v=3 v, T v=\sin v$. Clearly, $v_{1} \in \mathbb{R}, T$ is nonexpansive and $F(T)=\{0\}, \nabla, B$ and $\mathscr{F}$ satisfy the conditions of Theorems 3.1 and 3.2, respectively. The parameters $\lambda=0.04, \delta=0.01, \mu=0.335 . \lambda=0.05$ and $\delta=0.015$. The graph of numerical experiments are given below. The $y$-axis represents the values of $\left|v_{n}-0\right|$, while the $x$-axis represents the number of iterations $(n)$.

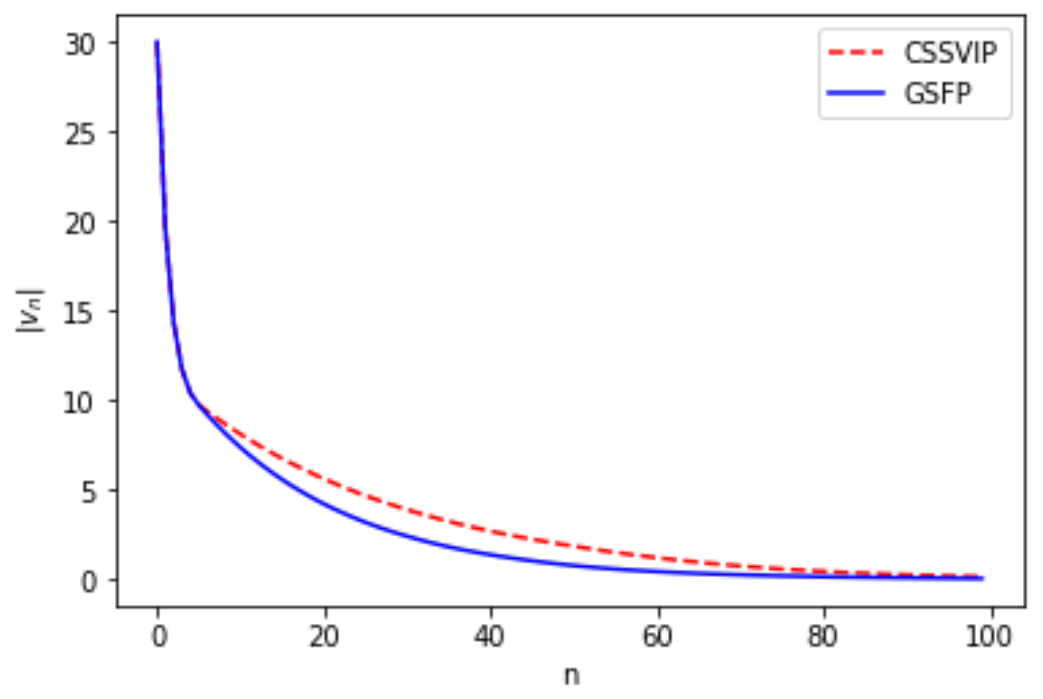

FIGURE 1. Numerical example with $v_{1}=30$ 


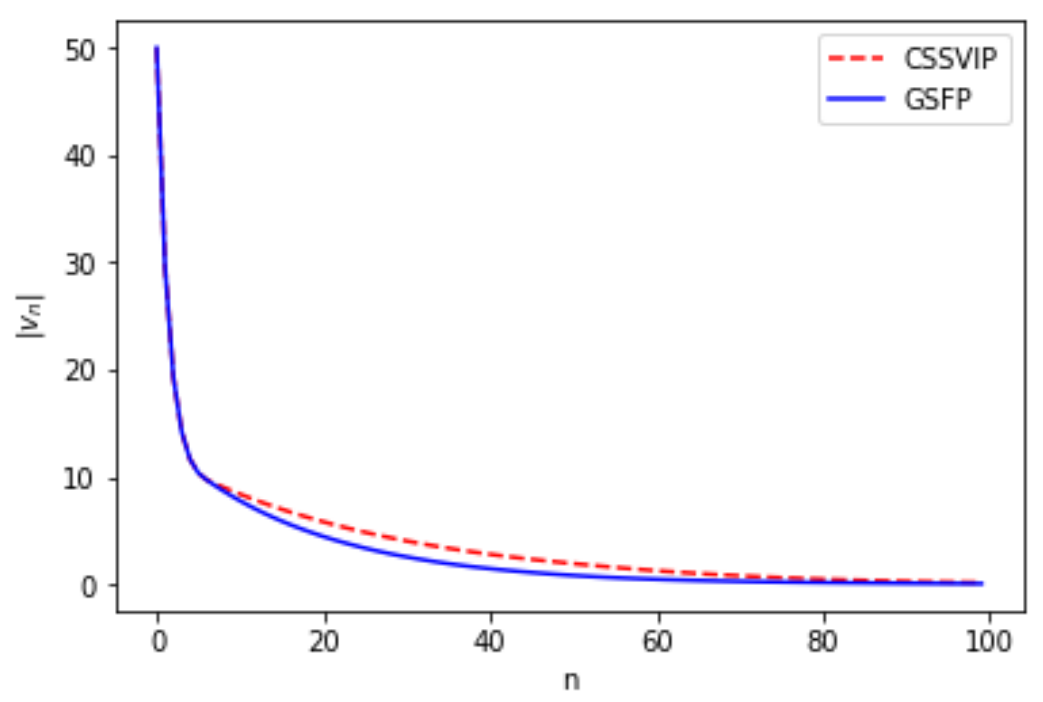

FIGURE 2. Numerical example with $v_{1}=50$

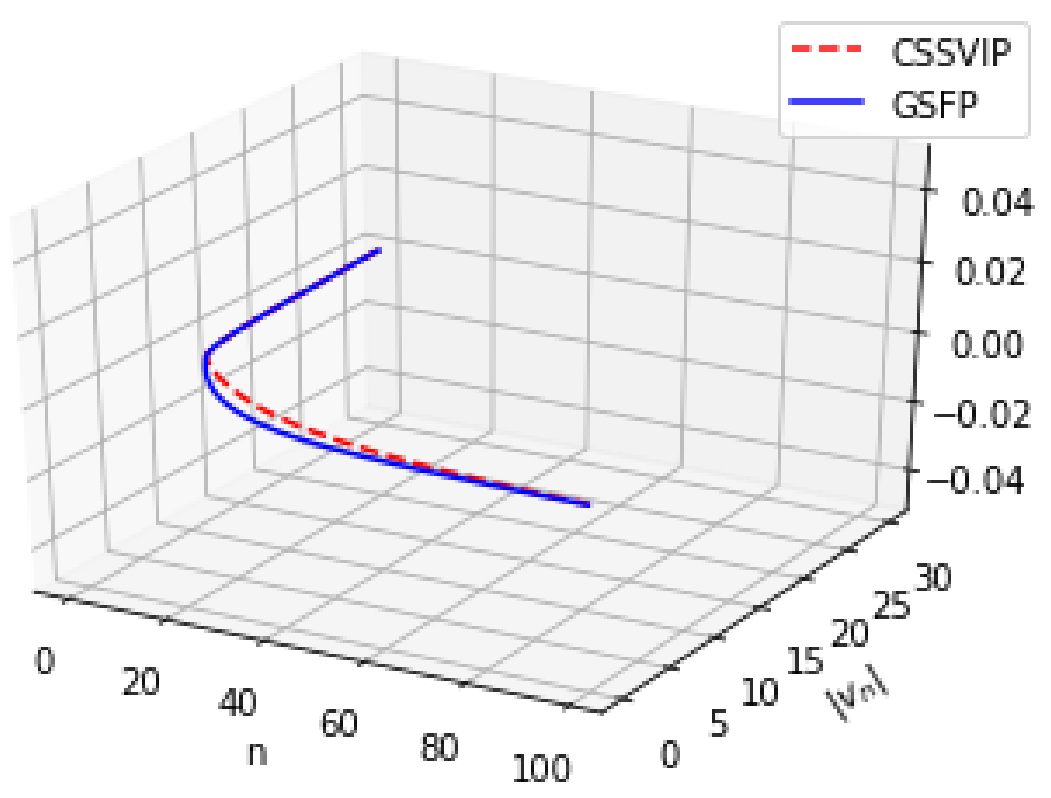

FIGURE 3. Numerical example with $v_{1}=50$

All computations and graphs were implemented in python 3.6 using some abstractions developed at the African University of Science and Technology and other open source python library such as numpy and matplotlib on Zinox with intel core $i 7$ process.

Discussion. Theorem 3.1 and Theorem 3.2 improve the recent results in Censor et al. [3], Tian and Jiang [19] as follows.

Even for a single operator, the algorithms in Theorems 3.1 and 3.2 are slightly different from the algorithms studied by Tian and Jiang [19]. 
(1) If $T_{i} \equiv P_{Q_{i}}=\left(I-\mu \mathscr{F}_{i}\right) \equiv 0, \nabla \equiv I$ and $\lambda \equiv \delta$, then, the (CSSVIP) is reduced to the (CSVIP) and equation (3.1) in Theorem 3.2 is reduced to the theorem of Censor et al. [3] for solving (CSVIP).

(2) Theorems 3.1 and 3.2 are strong convergence theorems while there are only weak convergence results in Tian and Jiang [19].

Finally, we remark that the Lipschitz constant $L>0$ and the norm $\|\nabla\|$ used in equations (3.1) and (3.18) of this paper and the norm $\|A\|$ used in the theorems of Tian and Jiang [19] need not to be known precisely. Any upper bound for these constants which are not difficult to obtain will do. Theorems 3.1 3.4 can not be proved if we use Halpern-type techniques in our algorithms.

\section{Acknowledgment}

This paper was supported by the ACBF Research Grant to African University of Science and Technology, Nigeria.

\section{REFERENCES}

[1] E. Blum, W. Oettli, From optimization and variational inequalities to equilibrium problems, Math. Stud. 63 (1994), 123145 .

[2] L.C. Ceng, A. Petrusel, J.C. Yao, Y. Yao, Hybrid viscosity extragradient method for systems of variational inequalities, fixed points of nonexpansive mappings, zero points of accretive operators in Banach spaces, Fixed Point Theory 19 (2018), 487-503.

[3] Y. Censor, A. Gibali, S. Reich, S. Sabach, Common solutions to variational inequalities, Set-Valued Var. Anal. 20 (2012), 229-247.

[4] Y. Censor, T. Elfving, A multi projection algorithm using Bregman projections in a product space, Numer. Algorithms 8 (1994), 221-239.

[5] Y. Censor A. Gibali, S. Reich, The split variational inequality problem, The Technion- Isreal Institute of Technology, Haifa, arXiv: 1009.3780 (2010).

[6] C.E. Chidume, A. Adamu, L. Okereke, A Krasnoselskii-type algorithm for approximating solutions of variational inequality problems and convex feasibility problems, J. Nonlinear Var. Anal. 2 (2018), 203-218.

[7] C.E. Chidume, L.O. Chiwendu, A. Adamu, A hybrid algorithm for approximating solutions of a variational inequality problem and a convex feasibility problem, Adv. Nonlinear Var. Inequal. 21 (2018), 46-64.

[8] P.L. Combettes, S.A. Hirstoaga, Equilibrium programming in Hilbert spaces, J. Nonlinear Convex Anal. 6 (2005), 117136.

[9] K. Goebel, S. Reich, Uniform Convexity, Hyperbolic Geometry, and Nonexpansive Mappings, Monographs and Textbooks in Pure and Applied Mathematics, Marcel Dekker, New York, (1984).

[10] D.V. Hieu, D.V. Thong, New extragradient-like algorithms for strongly pseudomonotone variational inequalities, 70 (2018), 385-399.

[11] G.M. Korpelevich, The extragradient method for finding saddle points and other problems, Ekon. Mat. Metody 12 (1976), 747-756.

[12] R. Kraikaew, S. Saejung, Strong convergence of the Halpern subgradient extragradient method for solving variational inequalities in Hilbert spaces, J. Optim. Theory Appl. 163 (2014), 399-412.

[13] P.D. Khanh, A new extragradient method for strongly pseudo-monotone variational inequalities, Numer. Funct. Anal. Optim. 37 (2016), 1131-1143.

[14] Y. Liu, A modified hybrid method for solving variational inequality problems in Banach spaces. J. Nonlinear Funct. Anal. 2017 (2017), Article ID 31.

[15] N. Nadezhkina, W. Takahashi, Strong convergence theorem by a hybrid method for nonexpansive mappings and Lipschitzcontinuous monotone mappings, SIAM J. Optim. 16 (2016), 1230-1241.

[16] R.T. Rockafellar, On the maximality of sums of nonlinear monotone operators, Trans. Amer. Math. Soc. 149 (1970), 75-88. 
[17] F. Tinti, Numerical solution for pseudomonotone variational inequality problems by extragradient methods, In: Giannessi F., Maugeri A. (eds) Variational Analysis and Applications, Nonconvex Optimization and Its Applications, vol 79. Springer, Boston, MA, (2005).

[18] W. Takahashi, H.K. Xu, J.C. Yao, Iterative methods for generalized split feasibility problems in Hilbert spaces, Set-Valued Var. Anal. 23 (2015), 205-221.

[19] M. Tian, B. Jiang, Weak convergence theorem for a class of split variational inequality problems and applications in a Hilbert space, J. Inequal. Appl. 2017 (2017), Article ID 123.

[20] H.K. Xu, Iterative methods for the split feasibility problem in infinite-dimensional Hilbert space, Inverse Probl. 26 (2010), Article ID 10518. 